\title{
Cytokines as determinants of disease and disease interactions
}

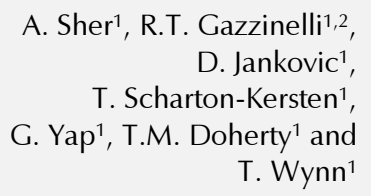

\author{
${ }^{1}$ Immunobiology Section, Laboratory of Parasitic Diseases, National Institute of Allergy \\ and Infectious Disease, National Institutes of Health, Bethesda, MD, USA \\ ${ }^{2}$ Departamento de Bioquímica e Imunologia, Universidade Federal de Minas Gerais, \\ Belo Horizonte, MG, Brasil
}

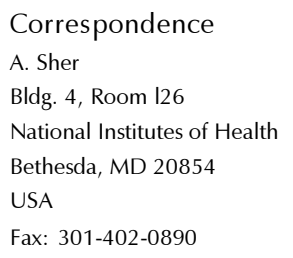

\section{Abstract}

The immune response to pathogens results in both host resistance and immunopathology. Cytokines and in particular those lymphokines produced by Th1 and Th2 cells play a key role in determining the balance between these two immunologic outcomes. Recent data suggest that interleukin-10, a product of both Th2 cells and macrophages, protects the host against excessive immunopathology. The cytokine environment generated by different pathogens may also influence the course and outcome of infections with unrelated organisms. This Key words - Cytokines - Disease - Immunopathology

- HIV-I

- Parasites

- Interleukin-10 relationship may be particularly important in the case of HIV-1 where prior Th1 or Th2 biases established by helminth or intracellular infections may influence either initial viral susceptibility or drive progression to AIDS through immune activation.

\section{Introduction}

The principal role of the immune system is thought to be host defense against invasion by pathogenic agents. For this reason, the study of the immunology of infection offers important insight concerning effector functions and regulatory interactions fundamental to the immune response. In reacting to infectious agents, the immune system can generate to varying degrees unwanted immunopathologic side effects in the form of fever, tissue damage and immune complex lesions. The balance between resistance and pathology is delicate and determined both by the virulence of the pathogen and the immunoregulatory state of the host. It has become increasingly clear that cytokines, and in particular those associated with the Th1/Th2 CD4+ T cell subsets, are key deter- minants of the beneficial vs disease consequence of the host response. Moreover, as discussed below, the characteristic pattern of cytokine expression triggered by one pathogen can influence the outcome of infections with unrelated infectious agents.

Regulation of host resistance and disease in the response to intracellular pathogens

Intracellular bacteria and protozoa usually stimulate and are controlled by type 1 (Th1) responses. Important examples include Listeria monocytogenes, Toxoplasma gondii, Mycobacterium tuberculosis and Leishmania major (in hosts with resistant genotypes). Nevertheless, the cytokines (e.g., IFN- $\gamma$, TNF$\alpha$, and IL-12) induced in the response to these agents can be quite toxic when pro- 
duced in large quantity. We postulate that IL-10, a cytokine whose synthesis is also stimulated by intracellular pathogens, plays a key role in protecting the host against excessive type 1 responsiveness. Thus, when infected with a normally avirulent strain of Toxoplasma gondii, IL-10 knockout mice rapidly succumb but with no evidence of increased parasite growth. Instead, these animals show extensive tissue necrosis associated with increased synthesis of IFN- $\gamma$, TNF$\alpha$, and IL-12 in either the circulation or from macrophage cultures (1). Thus, in the absence of IL-10 the balance is "tipped" and the normally protective immune response induced by $\mathrm{T}$. gondii becomes pathogenic (Figure 1).

Another scenario for the induction of pathology is the abortive attempt of the immune system to control intracellular pathogens resistant to type 1 effector mechanisms. The host response to mycobacteria presents a number of excellent examples of this phenomenon. In the case of mice infected with virulent strains of Mycobacterium avium, control of bacterial growth develops only late in chronic infection and is preceded and accompanied by increasing splenomegaly. Mice with defects in macrophage function (e.g., iNOS knockout mice) fail to develop this immunopathology yet display normal IFN- $\gamma$ control of infection (2). Thus, the type 1-dependent response to infection, while attempting to limit bacterial replication, induces disease and this process becomes chronic because of the failure to eliminate

Figure 1 - IL-10 protects against the host pathology induced by type 1 responses in intracellular infections. As suggested by this drawing, intracellular infections produce predominantly Th1 as opposed to Th2 cytokines and these are responsible for host resistance. IL-10 keeps this response "balanced" so that when it is removed host pathology ensues.

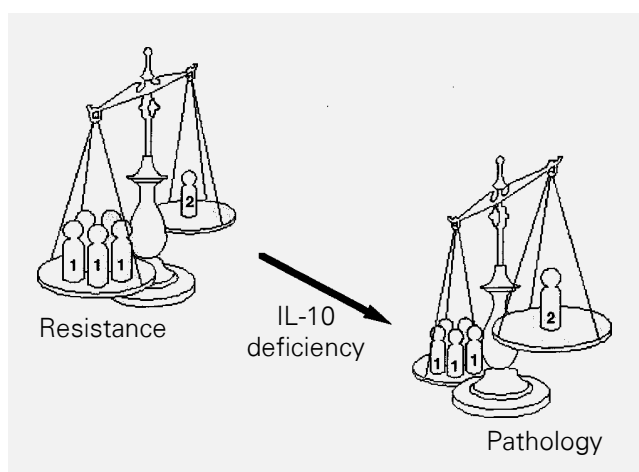

the microbial stimulus.

Regulation of Th2-associated pathogenesis in helminth infection

Helminth parasites induce responses which are predominantly $\mathrm{Th} 2$ in character. While there is debate as to whether these responses are important in host resistance, it is clear that they can contribute to tissue pathology in several worm-triggered diseases. In schistosomiasis, Th2 cytokines dictate granuloma formation in the intravenous egg injection model and contribute with the Th1 response to the pathogenesis of granulomas and tissue fibrosis in the livers of naturally infected mice. As in intracellular infection, IL-10 is strongly induced during both murine (3) and human schistosomiasis (4) and appears to be responsible for containing Th1 responses and in particular IFN$\gamma$ synthesis in lymphocyte cultures. Therefore, it was of interest to examine the effects of IL-10 deficiency on host pathology in this Th2-dominated disease. As expected, lymphocytes from egg-injected or S. mansoniinfected IL-10 knockout mice produced higher levels of IFN- $\gamma$ and reduced Th 2 cytokines than wild-type cells when stimulated with egg antigens in vitro. However, the effects on pathology differed dramatically with the site of the lesion. Thus, in IL-10 knockout mice, eggs injected into the lungs were smaller than in control animals (5) while eggs laid in the liver were greater in volume (Wynn T, Cheever AW, Williams ME, Hieny S, Caspar P, Kuhr R, Muller W \& Sher A, unpublished data). Therefore, in natural infection with this helminth, IL-10 appears to protect the host against excessive pathology, a function similar to its role in intracellular infection. Nevertheless, the mechanisms underlying this protection may be more complex than the simple regulation of Th1/Th2 function. For example, recent work suggests that $B$ cells may exert an even more profound effect on liver granuloma formation than Th1/Th2 cytokines (6). 
Influence of cytokines on susceptibility to infection with unrelated pathogens

The polarized cytokine environment induced by one pathogen can influence susceptibility and disease progression by unrelated infectious agents. Schistosome-infected mice show increased susceptibility to vaccinia (7), yet increased resistance to Trichuris infection (8), both effects associated with the enhanced production of Th2 and decreased synthesis of Th1 cytokines. Recent data on tetanus-vaccinated schistosome-infected patients indicate that a similar biasing of unrelated antigenic responses may occur in humans (9). In the opposite direction, intracellular bacterial and parasitic infections through their stimulation of TNF- $\alpha$ and other monokines can activate the HIV-1 virus and contribute to AIDS progression, a scenario which we have recently modeled in an experimental transgenic mouse system (10).

The above considerations have important implications for disease interactions at the populational level. For example, helminth-infected populations because of their decreased Th1 cytokine responses may be more susceptible to intracellular bacterial, parasitic and viral infections. In the case of HIV-1, this may translate into a loss in early cell-mediated control of the virus (11). On the other hand, intracellular infections such as $\mathrm{M}$. tuberculosis and $\mathrm{T}$. gondii could promote the rapid and often fatal AIDS progression seen in poorer countries by positively stimulating viral replication. Further study of these interactions and their cytokine dependencies may lead both to a better understanding of the pathogenesis of global infections and to the development of strategies for intervention.

\section{References}

1. Gazzinelli RT, Wysocka M, Hieny $S$, Scharton-Kersten T, Cheever A, Kuhn R, Muller W, Trinchieri G \& Sher A (1996). In the absence of endogenous IL-10 mice acutely infected with Toxoplasma gondii succumb to a lethal immune response dependent on CD4+ T cells and accompanied by overproduction of IL-12, IFN- $\gamma$ and TNF- $\alpha$. Journal of Immunology, 157: 798805.

2. Doherty TM \& Sher A (1997). Defects in cell-mediated immunity affect chronic, but not innate resistance of mice to Mycobacterium avium infection. Journal of Immunology, 158: 4822-4831.

3. Sher A, Fiorentino D, Caspar P, Pearce E \& Mosmann T (1991). Production of IL-10 by CD4+ T Iymphocytes correlates with down-regulation of Th1 cytokine synthesis in helminth infection. Journal of $1 \mathrm{~m}$ munology, 147: 2713-2716.

4. King CL, Medhat A, Malhotra I, Nafeh M, Helmy A, Khaudary J, Ibrahim S, ElSherbiny M, Zaky S, Stupi RJ, Brustoski K, Shehata M \& Shata MT (1996). Cytokine control of parasite-specific anergy in human urinary schistosomiasis. IL-10 modulates lymphocyte reactivity. Journal of Immunology, 156: 4715-4721.
5. Wynn TA, Morawetz R, Scharton-Kersten T, Hieny S, Morse III HC, Kuhn R \& Muller W (1998). Analysis of granuloma formation in double cytokine deficient mice reveals a central role for IL-10 in polarizing both Th1 and Th2-type cytokine responses in vivo. Journal of Immunology (in press).

6. Jankovic D, Kullberg M, Yap G, Epstein M, Cheever A \& Sher A (1996). B celldeficient mice exposed to Schistosoma mansoni develop exacerbated egg pathology which fails to down-modulate during chronic infection. FASEB Journal, 10: 1995 (Abstract).

7. Actor JK, Shirai M, Kullberg MC, Buller RM, Sher A \& Berzofsky JA (1993). Helminth infection results in decreased virusspecific CD8+ cytotoxic T-cell and Th1 cytokine responses as well as delayed virus clearance. Proceedings of the $\mathrm{Na}$ tional Academy of Sciences, USA, 90: 948-952.

8. Curry AJ, Else KJ, Jones F, Bancroft A, Grencis RK \& Dunne DW (1995). Evidence that cytokine-mediated immune interactions induced by Schistosoma mansoni alter disease outcome in mice concurrently infected with Trichuris muris. Journal of Experimental Medicine, 181: 769774.
9. Sabin EA, Araujo MI, Carvalho EM \& Pearce EJ (1996). Impairment of tetanus toxoid-specific Th1-like immune responses in humans infected with Schistosoma mansoni. Journal of Infectious Diseases, 173: 269-272.

10. Gazzinelli RT, Sher A, Cheever A, Gerstberger S, Martin MA \& Dickie P (1996). Infection of human immunodeficiency virus 1 transgenic mice with Toxoplasma gondii stimulates proviral transcription in macrophages in vivo. Journal of Experimental Medicine, 183: 16451655.

11. Clerici M \& Shearer GM (1994). Cellular immunity and a type 1 cytokine profile in protection against HIV infection and progression to AIDS. Research in Immunology, 145: 635-641. 